\title{
Why Information Security is Hard - An Economic Perspective
}

\author{
Ross Anderson \\ University of Cambridge Computer Laboratory, \\ JJ Thomson Avenue, Cambridge CB3 0FD, UK \\ Ross.Anderson@cl.cam.ac.uk
}

\begin{abstract}
According to one common view, information security comes down to technical measures. Given better access control policy models, formal proofs of cryptographic protocols, approved firewalls, better ways of detecting intrusions and malicious code, and better tools for system evaluation and assurance, the problems can be solved.
\end{abstract}

In this note, I put forward a contrary view: information insecurity is at least as much due to perverse incentives. Many of the problems can be explained more clearly and convincingly using the language of microeconomics: network externalities, asymmetric information, moral hazard, adverse selection, liability dumping and the tragedy of the commons.

\section{Introduction}

In a survey of fraud against autoteller machines [4], it was found that patterns of fraud depended on who was liable for them. In the USA, if a customer disputed a transaction, the onus was on the bank to prove that the customer was mistaken or lying; this gave US banks a motive to protect their systems properly. But in Britain, Norway and the Netherlands, the burden of proof lay on the customer: the bank was right unless the customer could prove it wrong. Since this was almost impossible, the banks in these countries became careless. Eventually, epidemics of fraud demolished their complacency. US banks, meanwhile, suffered much less fraud; although they actually spent less money on security than their European counterparts, they spent it more effectively [4].

There are many other examples. Medical payment systems that are paid for by insurers rather then by hospitals fail to protect patient privacy whenever this conflicts with the insurer's wish to collect information about its clients. Digital signature laws transfer the risk of forged signatures from the bank that relies on the signature (and that built the system) to the person alleged to have made the signature. Common Criteria evaluations are not made by the relying party, as Orange Book evaluations were, but by a commercial facility paid by the vendor. In general, where the party who is in a position to protect a system is not the party who would suffer the results of security failure, then problems may be expected.

A different kind of incentive failure surfaced in early 2000 , with distributed denial of service attacks against a number of high-profile web sites. These exploit a number of subverted machines to launch a large coordinated packet flood at a target. Since many of them flood the victim at the same time, the traffic is more than the target can cope with, and because it comes from many different sources, it can be very difficult to stop [7]. Varian pointed out that this was also a case of incentive failure [20]. While individual computer users might be happy to spend $\$ 100$ on anti-virus software to protect themselves against attack, they are unlikely to spend even $\$ 1$ on software to prevent their machines being used to attack Amazon or Microsoft.

This is an example of what economists refer to as the 'Tragedy of the Commons' [15]. If a hundred peasants graze their sheep on the village common, then whenever another sheep is added its owner gets almost the full benefit - while the other ninety-nine suffer only a small decline in the quality of the grazing. So they aren't motivated to object, but rather to add another sheep of their own and get as much of the grazing as they can. The result is a dustbowl; and the solution is regulatory rather than technical. A typical tenthcentury Saxon village had community mechanisms to deal with this problem; the world of computer security still doesn't. Varian's proposal is that the costs of distributed denial-of-service attacks should fall on the operators of the networks from which the flood- 
ing traffic originates; they can then exert pressure on their users to install suitable defensive software, or, for that matter, supply it themselves as part of the subscription package.

These observations prompted us to look for other ways in which economics and computer security interact.

\section{Network Externalities}

Economists have devoted much effort to the study of networks such as those operated by phone companies, airlines and credit card companies.

The more people use a typical network, the more valuable it becomes. The more people use the phone system - or the Internet - more people there are to talk to and so the more useful it is to each user. This is sometimes referred to as Metcalfe's law, and is not limited to communication systems. The more merchants take credit cards, the more useful they are to customers, and so the more customers will buy them; and the more customers have them, the more merchants will want to accept them. So while that networks can grow very slowly at first - credit cards took almost two decades to take off - once positive feedback gets established, they can grow very rapidly. The telegraph, the telephone, the fax machine and most recently the Internet have all followed this model.

As well as these physical networks, the same principles apply to virtual networks, such as the community of users of a mass-market software architecture. When software developers started to believe that the PC would outsell the Mac, they started developing their products for the PC first, and for the Mac only later (if at all). This effect was reinforced by the fact that the PC was easier for developers to work with. The growing volume of software available for the PC but not the Mac made customers more likely to buy a PC than a Mac, and the resulting positive feedback squeezed the Mac out of most markets. A similar effect made Microsoft Word the dominant word processor.

A good introduction to network economics is by Shapiro and Varian [17]. For our present purposes, there are three particularly important features of information technology markets.

- First, the value of a product to a user depends on how many other users adopt it.

- Second, technology often has high fixed costs and low marginal costs. The first copy of a chip or a software package may cost millions, but subsequent copies may cost very little to manufacture.
This isn't unique to information markets; it's also seen in business sectors such as airlines and hotels. In all such sectors, pure price competition will tend to drive revenues steadily down towards the marginal cost of production (which in the case of information is zero). So businesses need ways of selling on value rather than on cost.

- Third, there are often large costs to users from switching technologies, which leads to lock-in. Such markets may remain very profitable, even where (incompatible) competitors are very cheap to produce. In fact, one of the main results of network economic theory is that the net present value of the customer base should equal the total costs of their switching their business to a competitor [19].

All three of these effects tend to lead to "winner take all" market structures with dominant firms. So it is extremely important to get into markets quickly. Once in, a vendor will try to appeal to complementary suppliers, as with the software vendors whose bandwagon effect carried Microsoft to victory over Apple. In fact, successful networks tend to appeal to complementary suppliers even more than to users: the potential creators of "killer apps" need to be courted. Once the customers have a substantial investment in complementary assets, they will be locked in. (There are a number of complexities and controversies; see for example [14]. But the above simplified discussion will take us far enough for now.)

These network effects have significant consequences for the security engineer, and consequences that are often misunderstood or misattributed. Consultants often explain that the reason a design broke for which they were responsible was that the circumstances were impossible: 'the client didn't want a secure system, but just the most security I could fit on his product in one week on a budget of $\$ 10,000$ '. It is important to realize that this is not just management stupidity. The huge first-mover advantages that can arise in economic systems with strong positive feedback are the origin of the so-called "Microsoft philosophy" of 'we'll ship it on Tuesday and get it right by version 3'. Although sometimes attributed by cynics to a personal moral failing on the part of Bill Gates, this is perfectly rational behaviour in many markets where network economics apply.

Another common complaint is that software platforms are shipped with little or no security support, as with Windows 95/98; and even where access control mechanisms are supplied, as with Windows NT, they 
are easy for application developers to bypass. In fact, the access controls in Windows NT are often irrelevant, as most applications either run with administrator privilege (or, equivalently, require dangerously powerful operating system services to be enabled). This is also explained simply from the viewpoint of network economics: mandatory security would subtract value, as it would make life more difficult for the application developers. Indeed, Odlyzko observes that much of the lack of user-friendliness of both Microsoft software and the Internet is due to the fact that both Microsoft and the Internet achieved success by appealing to developers. The support costs that Microsoft dumps on users - and in fact even the cost of the time wasted waiting for PCs to boot up and shut down greatly exceed its turnover [16].

Network owners and builders will also appeal to the developers of the next generation of applications by arranging for the bulk of the support costs to fall on users rather than developers, even if this makes effective security administration impractical. One reason for the current appeal of public key cryptography may be that it can simplify development - even at the cost of placing an unreasonable administrative burden on users who are neither able nor willing to undertake it [9]. The technical way to try to fix this problem is to make security administration more 'user-friendly' or 'plug-and-play'; many attempts in this direction have met with mixed success. The more subtle approach is to try to construct an authentication system whose operators benefit from network effects; this is what Microsoft Passport does, and we'll discuss it further below.

In passing, it is worth mentioning that (thanks to distributed denial of service attacks) the economic aspects of security failure are starting to get noticed by government. A recent EU proposal recommends action by governments in response to market imperfections, where market prices do not accurately reflect the costs and benefits of improvemed network security [11]. However, this is only the beginning of the story.

\section{Competitive applications and corpo- rate warfare}

Network economics has many other effects on security engineering. Rather than using a standard, well analyzed and tested architecture, companies often go for a proprietary obscure one - to increase customer lock-in and increase the investment that competitors have to make to create compatible products. Where possible, they will use patented algorithms (even if these are not much good) as a means of imposing licensing conditions on manufacturers. For example, the DVD Content Scrambling System was used as a means of insisting that manufacturers of compatible equipment signed up to a whole list of copyright protection measures [5]. This may have come under severe pressure, as it could prevent the Linux operating system from running on next-generation PCs; but efforts to foist non-open standards continue in many applications from SDMI and CPRM to completely proprietary systems such as games consoles.

A very common objective is differentiated pricing. This is usually critical to firms that price a product or service not to its cost but to its value to the customer. This is familiar from the world of air travel: you can spend $\$ 200$ to fly the Atlantic in coach class, $\$ 2000$ in business class or $\$ 5000$ in first. Some commentators are surprised by the size of this gap; yet a French economist, Jules Dupuit, had already written in 1849:

[I]t is not because of the few thousand francs which would have to be spent to put a roof over the third-class carriage or to upholster the third-class seats that some company or other has open carriages with wooden benches . . . What the company is trying to do is prevent the passengers who can pay the second-class fare from traveling third class; it hits the poor, not because it wants to hurt them, but to frighten the rich. . - And it is again for the same reason that the companies, having proved almost cruel to the third-class passengers and mean to the second-class ones, become lavish in dealing with first-class customers. Having refused the poor what is necessary, they give the rich what is superfluous. [10]

This is a also common business model in the software and online services sectors. A basic program or service may be available free; a much better one for a subscription; and a 'Gold' service at a ridiculous price. In many cases, the program is the same except that some features are disabled for the budget user. Many cryptographic and other technical protection mechanisms have as their real function the maintenance of this differential.

Another business strategy is to manipulate switching costs. Incumbents try to increase the cost of switching, whether by indirect methods such as controlling marketing channels and building industries of complementary suppliers, or, increasingly, by direct 
methods such as making systems incompatible and hard to reverse engineer. Meanwhile competitors try to do the reverse: they look for ways to reuse the base of complementary products and services, and to reverse engineer whatever protection the incumbent builds in. This extends to the control of complementary vendors, sometimes using technical mechanisms.

Sometime, security mechanisms have both product differentiation and higher switching costs as goals. An example which may become politicized is 'accessory control'. According to one company that sells authentication chips into the automative market, some printer companies have begun to embed cryptographic authentication protocols in laser printers to ensure that genuine toner cartridges are used. If a competitor's cartridge is loaded instead, the printer will quietly downgrade from $1200 \mathrm{dpi}$ to $300 \mathrm{dpi}$. In mobile phones, much of the profit is made on batteries, and authentication can be used to spot competitors' products so they can be drained more quickly [3].

Another example comes from Microsoft Passport. This is a system whose ostensible purpose is single signon: a Passport user doesn't have to think up separate passwords for each participating web site, with all the attendant hassle and risk. Instead, sites that use Passport share a central authentication server run by Microsoft to which users log on. They use web redirection to connect their Passport-carrying visitors to this server; authentication requests and responses are passed back and forth by the user's browser in encrypted cookies. So far, so good.

But the real functions of Passport are somewhat more subtle [18]. First, by patching itself into all the web transactions of participating sites, Microsoft can collect a huge amount of data about online shopping habits and enable participants to swap it. If every site can exchange data with every other site, then the value of the network to each participating web site grows with the number of sites, and there is a strong network externality. So one such network may come to dominate, and Microsoft hopes to own it. Second, the authentication protocols used between the merchant servers and the Passport server are proprietary variants of Kerberos, so the web server must use Microsoft software rather than Apache or Netscape (this has supposedly been 'fixed' with the latest release, but participating sites still cannot use their own authentication server, and so remain in various ways at Microsoft's mercy).

So Passport isn't so much a security product, as a play for control of both the web server and purchasing information markets. It comes bundled with services such as Hotmail, is already used by 40 million people, and does 400 authentications per second on average. Its known flaws include that Microsoft keeps all the users' credit card details, creating a huge target; various possible middleperson attacks; and that you can be impersonated by someone who steals your cookie file. (Passport has a 'logout' facility that's supposed to delete the cookies for a particular merchant, so you can use a shared PC with less risk, but this feature didn't work properly for Netscape users when it was first deployed [13].)

The constant struggles to entrench or undermine monopolies and to segment and control markets determine many of the environmental conditions that make the security engineer's work harder. They make it likely that, over time, government interference in information security standards will be motivated by broader competition issues, as well as by narrow issues of the effectiveness of infosec product markets (and law enforcement access to data).

So much for commercial information security. But what about the government sector? As information attack and defense become ever more important tools of national policy, what broader effects might they have?

\section{Information Warfare - Offense and Defense}

One of the most important aspects of a new technology package is whether it favours offense or defense in warfare. The balance has repeatedly swung back and forth, with the machine gun giving an advantage to the defense in World War 1, and the tank handing it back to the offense by World War 2 .

The difficulties of developing secure systems using a penetrate-and-patch methodology have been known to the security community since at least the Anderson report in the early 1970s [2]; however, a new insight on this can be gained by using an essentially economic argument, that enables us to deal with vulnerabilities in a quantitative way [6].

To simplify matters, let us suppose a large, complex product such as Windows 2000 has 1,000,000 bugs, each with an MTBF of 1,000,000,000 hours. Suppose that Paddy works for the Irish Republican Army, and his job is to break into the British Army's computer to get the list of informers in Belfast; while Brian is the army assurance guy whose job is to stop Paddy. So he must learn of the bugs before Paddy does.

Paddy has a day job so he can only do 1000 hours of testing a year. Brian has full Windows source code, 
dozens of PhDs, control of the commercial evaluation labs, an inside track on CERT, an information sharing deal with other UKUSA member states - and he also runs the government's scheme to send round consultants to critical industries such as power and telecomms to advise them how to protect their systems. Suppose that Brian benefits from 10,000,000 hours a year worth of testing.

After a year, Paddy finds a bug, while Brian has found 100,000. But the probability that Brian has found Paddy's bug is only $10 \%$. After ten years he will find it - but by then Paddy will have found nine more, and it's unlikely that Brian will know of all of them. Worse, Brian's bug reports will have become such a firehose that Microsoft will have killfiled him.

In other words, Paddy has thermodynamics on his side. Even a very moderately resourced attacker can break anything that's at all large and complex. There is nothing that can be done to stop this, so long as there are enough different security vulnerabilities to do statistics: different testers find different bugs. (The actual statistics are somewhat more complicated, involving lots of exponential sums; keen readers can find the details at [6].)

There are various ways in which one might hope to escape this statistical trap.

- First, although it's reasonable to expect a 35,000,000 line program like Windows 2000 to have $1,000,000$ bugs, perhaps only $1 \%$ of them are security-critical. This changes the game slightly, but not much; Paddy now needs to recruit 100 volunteers to help him (or, more realistically, swap information in a grey market with other subversive elements). Still, the effort required of the attacker is still much less than that needed for effective defense.

- Second, there may be a single fix for a large number of the security critical bugs. For example, if half of them are stack overflows, then perhaps these can all be removed by a new compiler.

- Third, you can make the security critical part of the system small enough that the bugs can be found. This was understood, in an empirical way, by the early 1970s. However, the discussion in the above section should have made clear that a minimal TCB is unlikely to be available anytime soon, as it would make applications harder to develop and thus impair the platform vendors' appeal to developers.
So information warfare looks rather like air warfare looked in the 1920s and 1930s. Attack is simply easier than defense. Defending a modern information system could also be likened to defending a large, thinly-populated territory like the nineteenth century Wild West: the men in black hats can strike anywhere, while the men in white hats have to defend everywhere. Another possible relevant analogy is the use of piracy on the high seas as an instrument of state policy by many European powers in the sixteenth and seveteenth centuries. Until the great powers agreed to deny pirates safe haven, piracy was just too easy.

The technical bias in favour of attack is made even worse by asymmetric information. Suppose that you head up a U.S. agency with an economic intelligence mission, and a computer scientist working for you has just discovered a beautiful new exploit on Windows 2000. If you report this to Microsoft, you will protect 250 million Americans; if you keep quiet, you will be able to conduct operations against 400 million Europeans and 100 million Japanese. What's more, you will get credit for operations you conduct successfully against foreigners, while the odds are that any operations that they conduct successfully against U.S. targets will remain unknown to your superiors. This further emphasizes the motive for attack rather than defense. Finally - and this appears to be less widely realized - the balance in favour of attack rather than defense is still more pronounced in smaller countries. They have proportionally fewer citizens to defend, and more foreigners to attack.

In other words, the increasing politicization of information attack and defense may even be a destabilizing factor in international affairs.

\section{Distinguishing Good from Bad}

Since Auguste Kerckhoffs wrote his two seminal papers on security engineering in 1883 [12], people have discussed the dangers of 'security-by-obscurity', that is, relying on the attacker's being ignorant of the design of a system. Economics can give us a fresh insight into this. We have already seen that obscure designs are often used deliberately as a means of entrenching monopolies; but why is it that, even in relatively competitive security product markets, the bad products tend to drive out the good?

The theory of asymmetric information gives us an explanation of one of the mechanisms. Consider a used car market, on which there are 100 good cars (the 'plums'), worth $\$ 3000$ each, and 100 rather troublesome ones (the 'lemons'), each of which is worth only $\$ 1000$. The vendors know which is which, but the 
buyers don't. So what will be the equilibrium price of used cars?

If customers start off believing that the probability they will get a plum is equal to the probability they will get a lemon, then the market price will start off at $\$ 2000$. However, at that price only lemons will be offered for sale, and once the buyers observe this, the price will drop rapidly to $\$ 1000$ with no plums being sold at all. In other words, when buyers don't have as much information about the quality of the products as sellers do, there will be severe downward pressure on both price and quality. Infosec people frequently complain about this in many markets for the products and components we use; the above insight, due to Akerlof [1], explains why it happens.

The problem of bad products driving out good ones can be made even worse when the people evaluating them aren't the people who suffer when they fail. Much has been written on the ways in which corporate performance can be adversely affected when executives have incentives at odds with the welfare of their employer. For example, managers often buy products and services which they know to be suboptimal or even defective, but which are from big name suppliers. This is known to minimize the likelihood of getting fired when things go wrong. Corporate lawyers don't condemn this as fraud, but praise it as 'due diligence'. Over the last decade of the twentieth century, many businesses have sought to fix this problem by extending stock options to ever more employees. However, these incentives don't appear to be enough to ensure prudent practice by security managers. (This might be an interesting topic for a $\mathrm{PhD}$; does it come down to the fact that security managers also have less information about threats, and so cannot make rational decisions about protection versus insurance, or is it simply due to adverse selection among security managers?)

This problem has long been perceived, even if not in precisely these terms, and the usual solution to be proposed is an evaluation system. This can be a private arrangement, such as the equipment tests carried out by insurance industry laboratories for their member companies, or it can be public sector, as with the Orange Book and the Common Criteria.

For all its faults, the Orange Book had the virtue that evaluations were carried out by the party who relied on them - the government. The European equivalent, ITSEC, introduced a pernicious innovation - that the evaluation was not paid for by the government but by the vendor seeking an evaluation on its product.
This got carried over into the Common Criteria.

This change in the rules provided the critical perverse incentive. It motivated the vendor to shop around for the evaluation contractor who would give his product the easiest ride, whether by asking fewer questions, charging less money, taking the least time, or all of the above. To be fair, the potential for this was realized, and schemes were set up whereby contractors could obtain approval as a CLEF (commercial licensed evaluation facility). The threat that a CLEF might have its license withdrawn was supposed to offset the commercial pressures to cut corners.

But in none of the half-dozen or so disputed cases I've been involved in has the Common Criteria approach proved satisfactory. Some examples are documented in my book, Security Engineering [3]. The failure modes appear to involve fairly straightforward pandering to customers' wishes, even (indeed especially) where these were in conflict with the interests of the users for whom the evaluation was supposedly being prepared. The lack of sanctions for misbehaviour - such as a process whereby evaluation teams can lose their accreditation when they lose their sparkle, or get caught in gross incompetence or dishonesty, is probably a contributory factor.

But there is at least one more significant perverse incentive. From the user's point of view, an evaluation may actually subtract from the value of a product. For example, if you use an unevaluated product to generate digital signatures, and a forged signature turns up which someone tries to use against you, you might reasonably expect to challenge the evidence by persuading a court to order the release of full documentation to your expert witnesses. A Common Criteria certificate might make a court much less ready to order disclosure, and thus could severely prejudice your rights. A cynic might suggest that this is precisely why it's the vendors of products which are designed to transfer liability (such as digital signature smartcards), to satisfy due diligence requirements (such as firewalls) or to impress naive users (such as PC access control products), who are most enthusiastic about the Common Criteria.

So an economist is unlikely to place blind faith in a Common Criteria evaluation. Fortunately, the perverse incentives discussed above should limit the uptake of the Criteria to sectors where an official certification, however irrelevant, erroneous or misleading, offers competitive advantage. 


\section{Conclusions}

Much has been written on the failure of information security mechanisms to protect end users from privacy violations and fraud. This misses the point. The real driving forces behind security system design usually have nothing to do with such altruistic goals. They are much more likely to be the desire to grab a monopoly, to charge different prices to different users for essentially the same service, and to dump risk. Often this is perfectly rational.

In an ideal world, the removal of perverse economic incentives to create insecure systems would depoliticize most issues. Security engineering would then be a matter of rational risk management rather than risk dumping. But as information security is about power and money - about raising barriers to trade, segmenting markets and differentiating products - the evaluator should not restrict herself to technical tools like cryptanalysis and information flow, but also apply economic tools such as the analysis of asymmetric information and moral hazard. As fast as one perverse incentive can be removed by regulators, businesses (and governments) are likely to create two more.

In other words, the management of information security is a much deeper and more political problem than is usually realized; solutions are likely to be subtle and partial, while many simplistic technical approaches are bound to fail. The time has come for engineers, economists, lawyers and policymakers to try to forge common approaches.

\section{Acknowledgements}

I got useful comments on early drafts of some of this material from Avi Rubin, Hal Finney, Jack Lang, Andrew Odlyzko and Hal Varian.

\section{Postscript}

The dreadful events of September 11th happened just before this manuscript was finalised. They will take some time to digest, and rather than rewriting the paper it seemed better to add this short postscript.

I believe that the kind of economic arguments advanced here will be found to apply to protecting 'bricks' as much as 'clicks'. It may take years for the courts to argue about liability; there will remain a strong public interest in ensuring that the operational responsibility for protection does not become divorced from the liability for the failure of that protection.

The arguments in section 4 are also brought into sharper relief. In a world in which the 'black hats' can attack anywhere but the 'white hats' have to defend everywhere, the black hats have a huge economic advantage. This suggests that local defensive protection is not enough; there is an essential role for global defence, of which deterrence and retribution may be an unavoidable part.

The suppression of piracy, mentioned in that section, may be a useful example. It might also be a sobering one. Although, from the late seventeenth century, major governments started to agree that the use of pirates as instruments of state policy was unacceptable, there was no single solution. It took many treaties, many naval actions, and the overthrow of a number of rogue governments, over a period of more than a century, to pacify the world's oceans. The project became entwined, in complex ways, with other campaigns, including the abolition of slavery and the spread of colonialism. Liberals faced tough moral dilemmas: was it acceptable to conquer and colonise a particular territory, in order to suppress piracy and slavery there? In the end, economic factors appear to have been politically decisive; piracy simply cost business too much money. History may not repeat itself, but it might not be wise to ignore it.

\section{References}

[1] GA Akerlof, "The Market for 'Lemons': Quality Uncertainty and Market Mechanism," Quarterly Journal of Economics v 84 (August 1970) pp 488-500

[2] J Anderson, 'Computer Security Technology Planning Study', ESD-TR-73-51, US Air Force Electronic Systems Division (1973) http://csrc.nist.gov/publications/ history/index.html

[3] RJ Anderson, 'Security Engineering - A Guide to Building Dependable Distributed Systems', Wiley (2001) ISBN 0-471-38922-6

[4] RJ Anderson, "Why Cryptosystems Fail" in Communications of the ACM vol 37 no 11 (November 1994) pp 32-40

[5] JA Bloom, IJ Cox, T Kalker, JPMG Linnartz, ML Miller, CBS Traw, "Copy Protection for DVD Video", in Proceedings of the IEEE v 87 no 7 (July 1999) pp 1267-1276

[6] RM Brady, RJ Anderson, RC Ball, 'Murphy's law, the fitness of evolving species, and the limits of software reliability', Cambridge University Computer Laboratory Technical Report 
no. 476 (1999); at http;//www.cl.cam.ac. $\mathrm{uk} /{ }^{\sim} \mathrm{rja}$ 14

[7] CERT, Results of the Distributed-Systems Intruder Tools Workshop, Software Engineering Institute, Carnegie Mellon University, http://www.cert.org/reports/dsit_ workshop-final.html, December 7, 1999

[8] W Curtis, H Krasner, N Iscoe, "A Field Study of the Software Design Process for Large Systems", in Communications of the $A C M \mathrm{v} 31$ no 11 (Nov 88) pp 1268-1287

[9] D Davis, "Compliance Defects in Public-Key Cryptography", in Sixth Usenix Security Symposium Proceedings (July 1996) pp 171-178

[10] "De l'influence des péages sur l'utilité des voies de communication", 1849, Annales des Ponts et Chausses.

[11] European Union, 'Network and Information Security: Proposal for a European Policy Approach', $\operatorname{COM(2001)298~final,~6/6/2001~}$

[12] A Kerckhoffs, "La Cryptographie Militaire", in Journal des Sciences Militaires, 9 Jan 1883, pp 5-38; http://www.fabien-petitcolas.net/ kerckhoffs/

[13] DP Kormann, AD Rubin, "Risks of the Passport Single Signon Protocol", in Computer Networks (July 2000); at http://avirubin. com/vita.html

[14] SJ Liebowitz, SE Margolis, "Network Externalities (Effects)", in The New Palgrave's Dictionary of Economics and the Law, MacMillan, 1998; see http: //wwwpub.utdallas.edu/ riebowit/netpage.html

[15] WF Lloyd, 'Two Lectures on the Checks to Population', Oxford University Press (1833)

[16] AM Odlyzko, "Smart and stupid networks: Why the Internet is like Microsoft", ACM netWorker, Dec 1998, pp 38-46, at http://www.acm.org/networker/ issue/9805/ssnet.html

[17] C Shapiro, H Varian, 'Information Rules', Harvard Business School Press (1998), ISBN 0-87584-863-X
[18] J Spolsky, "Does Issuing Passports Make Microsoft a Country?" at http://joel.editthispage.com/stories/ storyReader $\$ 139$

[19] H Varian, 'Intermediate Microeconomics - A Modern Approach', Fifth edition, WW Norton and Company, New York, 1999; ISBN 0-39397930-0

[20] H Varian, "Managing Online Security Risks", Economic Science Column, The New York Times, June 1, 2000, http: //www . nytimes.com/library/financial/ columns/060100econ-scene.html 